Ophthalmologica

Ophthalmologica 2010;224:59-62

DOI: 10.1159/000235727
Received: July 31, 2008

Accepted after revision: January 23, 2009

Published online: August 26, 2009

\title{
Intracellular Extraocular Muscle Light- and Heavy-Chain Deposition Disease Contributing to Compressive Optic Neuropathy in a Patient with Preexisting Graves' Orbitopathy
}

\author{
Peter A. Zakrzewski ${ }^{a}$ Ezekiel Weis ${ }^{b}$ Valerie A. White ${ }^{a}$ Jack Rootman ${ }^{a}$ \\ ${ }^{a}$ Department of Ophthalmology, University of British Columbia, Vancouver, B.C., and ${ }^{b}$ Department of \\ Ophthalmology, University of Alberta, Edmonton, Alta., Canada
}

\section{Key Words}

Monoclonal immunoglobulin deposition disease •

Light- and heavy-chain deposition disease $\cdot$ Compressive optic neuropathy $\cdot$ Graves' orbitopathy $\cdot$ Multiple myeloma

\begin{abstract}
Purpose: Light-and heavy-chain deposition disease (LHCDD) is a rare form of nonamyloidal monoclonal immunoglobulin deposition disease (MIDD) in which light- and heavy-chain immunoglobulin fragments accumulate systemically, typically leading to end organ dysfunction. Herein we describe the case of a 64-year-old female with a history of Graves' orbitopathy and multiple myeloma who presented with bilateral asymmetric compressive optic neuropathies. Procedure: A biopsy of the right medial rectus muscle was taken during orbital decompression surgery. Results: Light and electron microscopy of the biopsy specimen led to a diagnosis of intracellular skeletal muscle LHCDD. Conclusion: This is the first published report to describe the findings of: (1) intracellular deposition of nonamyloidal MIDD; (2) orbital involvement of nonamyloidal MIDD, and (3) compressive optic neuropathy resulting from any form of MIDD.
\end{abstract}

Copyright $\odot 2009$ S. Karger AG, Base

\section{KARGER}

Fax +41 613061234 E-Mail karger@karger.ch www.karger.com
(C) 2009 S. Karger AG, Basel

0030-3755/10/2241-0059\$26.00/0

Accessible online at:

www.karger.com/oph

\section{Case Report}

In 2006, a 64-year-old woman presented with gradually decreasing vision OD $>$ OS. Her medical history included multiple myeloma, Graves' hyperthyroidism, paroxysmal atrial flutter, chronic obstructive pulmonary disease, osteoporosis, pernicious anemia and thrombocytopenia. In 1993, she developed bilateral Graves' orbitopathy (GO), which did not require medical or surgical intervention.

On examination, visual acuities were counting fingers $\mathrm{OD}$ and $20 / 60$ OS. Color vision was decreased OD > OS with a right relative afferent papillary defect. There were bilaterally decreased eye movements and increased exophthalmos (Hertel exophthalmometry: $27 \mathrm{~mm}$ OD, $28 \mathrm{~mm}$ OS) compared with her most recent examination in 2004 (Hertel exophthalmometry: $21 \mathrm{~mm}$ OD, $20 \mathrm{~mm}$ OS). A CT scan of the orbits (fig. 1) revealed bilateral thickening and enhancement of the medial, superior and inferior rectus muscles and tendons, as well as the right lateral rectus muscle and tendon. Radiolucent areas were present within the bellies of these rectus muscles bilaterally, consistent with the presence of fat. There was bilateral $(\mathrm{R}>\mathrm{L})$ apical crowding, in keeping with an $\mathrm{R}>\mathrm{L}$ compressive optic neuropathy. The CT scan also demonstrated optic nerve sheath thickening and enhancement and enhancing infiltrates within the orbital fat, bilaterally. Comparative CT orbital imaging from 2001 (most recent imaging available) demonstrated a similar pattern of bilateral extraocular muscle thickening, but to a significantly lesser degree, without apical compression, muscle tendon thickening or optic nerve sheath enhancement. Serum protein analysis revealed monoclonal IgG heavy chains and monoclonal IgG $\lambda$ light chains. Renal, liver and thyroid testing (thyroid-stimulating hormone $2.81 \mathrm{mU} / \mathrm{l}$, free thyroxine
Peter Zakrzewski, MD

Department of Ophthalmology

2550 Willow Street

Vancouver, BC V5Z 3N9 (Canada)

Tel. +1 604875 4044, Fax +1 604875 5532, E-Mail pz@interchange.ubc.ca 
Fig. 1. Noncontrast CT scan of the orbits. a An axial scan demonstrates bilateral thickened medial rectus muscles (white arrowheads), bilateral optic nerve sheath enhancement (white arrow), right-sided apical crowding (black arrow) and multiple small enhancing areas within the orbital fat. b A coronal scan demonstrates bilateral superior (black arrowheads), medial (white arrowheads) and inferior (black arrows) rectus muscle thickening, and bilateral optic nerve sheath enhancement (white arrows).
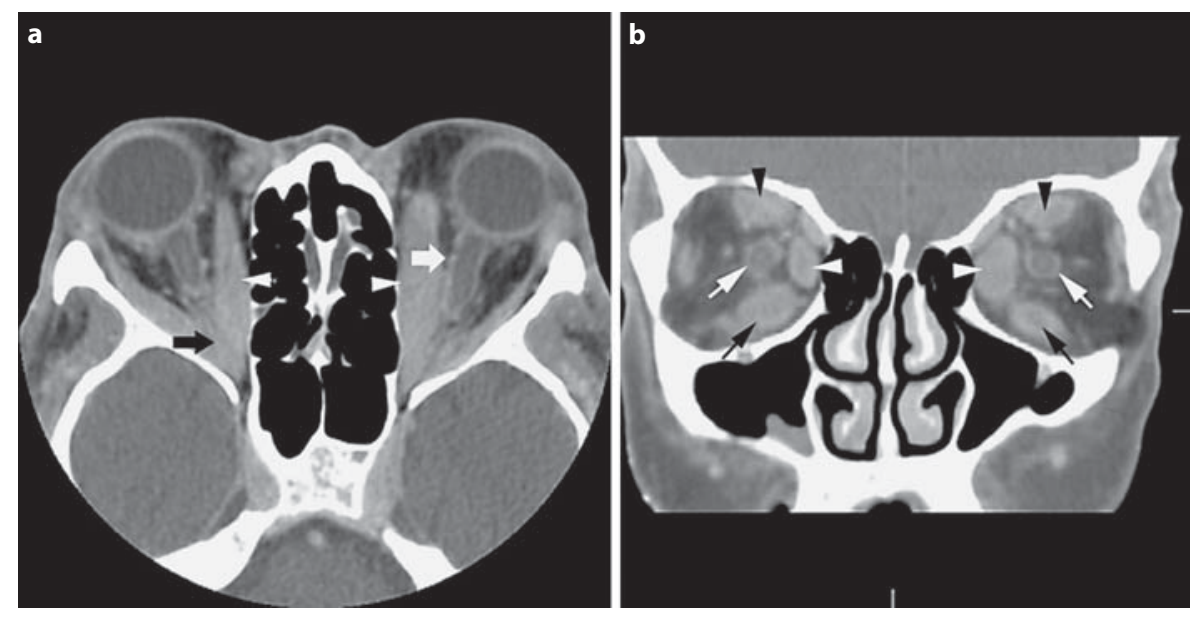

$15.5 \mathrm{pmol} / \mathrm{l}$, free triiodothyronine $2.81 \mathrm{pmol} / \mathrm{l}$ ) were all normal. An echocardiogram demonstrated mild diastolic dysfunction.

Pulse intravenous methylprednisolone (1 $\mathrm{g}$ daily for 3 days) provided initial, although incomplete, improvement to 20/300 OD and $20 / 50$ OS, suggesting that the inflammatory component made a small contribution, but the main factor was a change in mass. This was supported by vision subsequently deteriorating over 1 week to hand motions OD and 20/60 OS, and by the failure of the subsequently administered orbital radiotherapy $(2,000 \mathrm{cGy}$ over 10 fractions) to improve vision. The lack of a more significant and longerlasting improvement from the pulse corticosteroids and radiotherapy, as well as the CT features, was felt to be atypical of a case of GO.

Urgent bilateral orbital decompression surgery (medial and inferior orbital wall infracturing) was performed in an attempt to alleviate the compressive optic neuropathies. Although a recurrence of GO was considered a possible diagnosis based on clinical signs and symptoms, the history of multiple myeloma, lack of expected visual improvement with corticosteroids and radiotherapy, and atypical imaging features not present on older orbital imaging (extraocular muscle tendon thickening, optic nerve sheath enhancement, enhancing infiltrates within the orbital fat) led to the consideration of other causes, including infiltrative multiple myeloma, monoclonal immunoglobulin deposition disease (MIDD) and other inflammatory conditions. In order to elucidate the underlying cause of the optic neuropathy, a right medial rectus muscle biopsy was taken at the time of decompression.

Following the surgical decompression, visual acuities improved to $20 / 50 \mathrm{OU}$, which remained stable at 1 month of follow-up. No further follow-up was obtained as the patient was unable to attend subsequent clinic appointments. She died 6 months following the orbital surgery due to a cardiac arrest secondary to a presumed pulmonary embolism, although no autopsy was performed.

\section{Materials and Methods}

A right medial rectus muscle biopsy was obtained during orbital decompression surgery. The specimen was examined by light and electron microscopy using standard methods.

\section{Results}

Light-microscopic examination of the biopsy specimen (fig. $2 \mathrm{a}-\mathrm{d}$ ) revealed numerous amorphous, eosinophilic globules within the sarcolemma of extraocular skeletal muscle fibers. There were no lymphocytes or edema between muscle fibers, features that would be expected in active GO. Affected muscle fibers appeared swollen in size compared with adjacent unaffected fibers. The amorphous globules stained with periodic acid-Schiff and did not stain for actin and desmin, suggesting that they were not composed of muscle proteins. Masson trichrome staining demonstrated mature-looking fibroblasts and dense collagenous fibrosis between muscle fibers. Alcian blue staining for glycosaminoglycans was negative. Overall, the biopsy findings of a lack of lymphocytes, edema and glycosaminoglycans, along with the presence of extensive fibrosis and inactive fibroblasts, are more consistent with old GO without significant reactivation. There was no staining with Congo red, ruling out an amyloid component. By immunoperoxidase staining, the globules stained for IgG heavy chains and $\lambda$ light chains, and were negative for IgM and IgA heavy chains, and $\kappa$ light chains. Electron microscopy (fig. 2e, f) confirmed the presence of amorphous, dense globules, consistent with proteinaceous material, within the cytoplasm of the skeletal muscle fibers. Based on these histological findings, a diagnosis of light- and heavy-chain deposition disease (LHCDD) was made, with the bilateral compressive optic neuropathies the result of extraocular muscle volume expansion at the orbital apices from deposited LHCDD material superimposed upon preexisting fibrotic muscle thickening from GO. 
Fig. 2. Photomicrographs of right medial rectus muscle biopsy. a Numerous eosinophilic globules (black arrows) within the sarcolemma of some muscle fibers resulting in swelling of these fibers compared with nonaffected adjacent muscle fibers (white arrowheads), muscle regeneration evident by the enlarged, central nuclei (black arrowheads) and scattered inactive, mature-looking fibroblasts between muscle fibers. HE. $\times 400$. b Positive staining for dense collagenous fibrosis (blue in the online version) between muscle fibers. Masson trichrome. $\times 200$. c Positive staining for intracellular IgG heavy chains (white arrows). Anti-IgG-heavy-chain immunoperoxidase stain, hematoxylin counter-stain. $\times 400$. d Positive staining for intracellular $\lambda$ light chains (white arrowheads). Anti- $\lambda$-light-chain immunoperoxidase stain, hematoxylin counterstain. $\times 400$. e, $\mathbf{f}$ Transmission electron micrographs demonstrating numerous amorphous intracellular globules, consistent with protein (black arrows) within the muscle fiber cytoplasm. e $\times 6,300$. f $\times 10,000$.
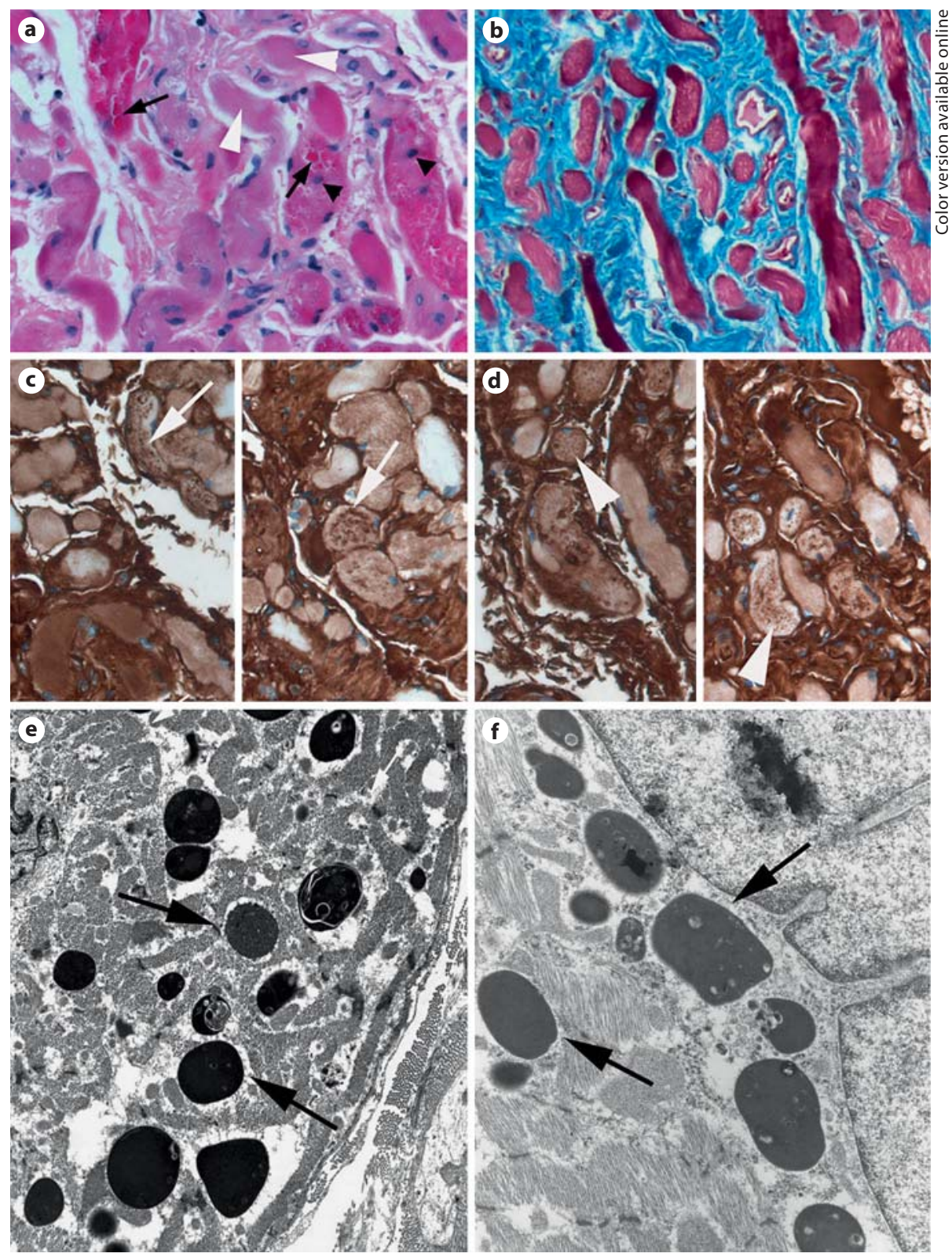

\section{Discussion}

MIDD represents a group of disorders in which systemic deposition and accumulation of abnormal immunoglobulins result in compromised end organ function. MIDD can be broken down into amyloidal and nonamyloidal subtypes. The amyloidal group consists of primary amyloidosis and heavy-chain amyloidosis, while the nonamyloidal group consists of light-chain deposition disease, LHCDD and heavy-chain deposition disease. In amyloidal MIDD, abnormal light or heavy chains with a $\beta$-pleated sheet structure form fibrillar, Congo-red-binding deposits in various tissues [1]. In the nonamyloidal forms, systemic deposition of abnormal light chains, heavy chains or both occurs, but the deposits appear granular or amorphous, and do not bind Congo red or form $\beta$-pleated sheets $[1,2]$. The deposits found in the medial rectus biopsy of our patient exhibited the appropriate features of nonamyloidal MIDD and contained both IgG heavy chains and $\lambda$ light chains, leading to the diagnosis of LHCDD. 
Light-chain deposition disease is the most common form of nonamyloidal MIDD, with the LHCDD subtype comprising less than $10 \%$ of reported cases [3]. For both amyloidal and nonamyloidal MIDD, the most commonly affected tissue sites are the kidney, heart and liver, frequently leading to organ compromise $[1,3]$. Multiple myeloma is present in $50-60 \%$ of nonamyloidal MIDD patients [3]. Serum and urine protein analysis often detect the presence of a monoclonal immunoglobulin in patients with MIDD [1]. In our patient, the immunoglobulin types found in her biopsy specimen, IgG heavy chains and $\lambda$ light chains, were the same as those measured in her serum.

There are several reports of nonamyloidal MIDD involving skeletal muscle; however, ours is the first to describe skeletal muscle involvement (extraocular muscle) in LHCDD. Rott et al. [4] describe the findings of IgG heavy-chain deposits in a skeletal muscle biopsy from a patient with heavy-chain deposition disease. The deposits were located in the endomysium along sarcolemmas, in vessel walls and in the perimysium. These locations are typical of MIDD as deposition most often occurs along basement membranes of targeted tissues, suggesting that the proteins likely have an affinity for some membrane component [1]. Ours is the first published report to document a case of nonamyloidal MIDD with intracellular skeletal muscle deposition.

This is also the first published report describing nonamyloidal MIDD involving orbital tissues, as well as the first to report any type of MIDD leading to compressive optic neuropathy. There are 2 published cases of orbital primary amyloidosis requiring orbital decompression surgery, but neither exhibited preoperative compressive optic neuropathy $[5,6]$.

In conclusion, although rare, MIDD (both amyloidal and nonamyloidal forms) should be considered in the differential diagnosis of orbital infiltrative diseases, especially if there is a history of multiple myeloma or atypical findings of the optic nerve sheath, rectus muscle and/or orbital fat enhancement on orbital imaging.

\section{References}

Buxbaum J, Gallo G: Nonamyloidotic monoclonal immunoglobulin deposition disease: light-chain, heavy-chain, and light- and heavy-chain deposition diseases. Hematol Oncol Clin North Am 1999;13:1235-1248.

-2 KhamlichiAA, Aucouturier P, Preud'homme JL, Cogné M: Structure of abnormal heavy chains in human heavy-chain-deposition disease. Eur J Biochem 1995;229:54-60.
Buxbaum JN, Chuba JV, Hellman GC, et al: Monoclonal immunoglobulin deposition disease: light chain and light and heavy chain deposition diseases and their relation to light chain amyloidosis: clinical features, immunopathology, and molecular analysis. Ann Intern Med 1990;112:455-464.

4 Rott T, Vizjak A, Lindic J, et al: IgG heavychain deposition disease affecting kidney, skin, and skeletal muscle. Nephrol Dial Transplant 1998;13:1825-1828.
5 Hamidi Asl K, Liepnieks JJ, Nunery WR, et al: Kappa III immunoglobulin light chain origin of localized orbital amyloidosis. Amyloid 2004;11:179-183.

6 Pasternak S, White VA, Gascoyne RD, et al: Monoclonal origin of localised orbital amyloidosis detected by molecular analysis. $\mathrm{Br} \mathrm{J}$ Ophthalmol 1996;80:1013-1017. 